\title{
Dipeptidyl peptidase IV (DPP-4) inhibition alleviates pulmonary arterial remodeling in experimental pulmonary hypertension
}

\author{
Jian $\mathrm{Xu}^{1} \cdot$ Jingjing Wang ${ }^{2} \cdot$ Mengyu $\mathrm{He}^{1} \cdot$ Honghao Han ${ }^{1} \cdot$ Weiping $\mathrm{Xie}^{1} \cdot \mathrm{Hong}$ Wang $\mathbb{D}^{1} \cdot$ Hui Kong ${ }^{1}$
}

Received: 18 November 2017 / Revised: 3 April 2018 / Accepted: 19 April 2018 / Published online: 22 May 2018

(c) United States \& Canadian Academy of Pathology 2018

\begin{abstract}
Dipeptidyl peptidase IV (DPP-4) is well known for its role in glucose homeostasis, and DPP-4 inhibitor (DPP-4i) exhibits multiple actions in cardiovascular diseases. However, the effect of DPP-4i on pulmonary hypertension (PH) remains unclear. Therefore, this study aims to investigate the effect of DPP-4i on pulmonary arterial remodeling in rats with PH and the potential underlying mechanisms. Our results show that DPP-4 was expressed in epithelial cells, endothelial cells, smooth muscle cells, and inflammatory cells in lung. DPP-4i (Sitagliptin) attenuated right ventricular systolic pressure (RVSP), right ventricle remodeling, hypertrophy of pulmonary arterial medial layer, inflammatory cell infiltration, and endothelial-mesenchymal transition (EndMT) in monocrotaline (MCT)-induced PH rats. Similarly, DPP-4i also alleviated bleomycin- and chronic hypoxia-induced PH in rats. In cultured human pulmonary arterial smooth muscle cells (PASMCs), DPP-4i inhibited platelet derived growth factor (PDGF)-BB-induced proliferation and migration, which was abolished by phosphatase and tensin homolog deleted on chromosome ten (PTEN) knockout. These results demonstrate that DPP-4 inhibition alleviates pulmonary arterial remodeling in experimental $\mathrm{PH}$ by inhibiting proliferation and migration of PASMCs.
\end{abstract}

\section{Introduction}

Knowledge of the pathogenesis of pulmonary hypertension $(\mathrm{PH})$ has been evolving. Alterations in mitochondrial structure, disordered metabolism, inflammation, and increased growth factors lead to a hyperproliferative, apoptosis-resistant state of pulmonary arterial smooth

These authors contributed equally: Jian Xu, Jingjing Wang.

Electronic supplementary material The online version of this article (https://doi.org/10.1038/s41374-018-0080-1) contains supplementary material, which is available to authorized users.

\section{Hong Wang}

hongwang@njmu.edu.cn

$\triangle$ Hui Kong

konghui@njmu.edu.cn

1 Department of Respiratory and Critical Care Medicine, The First Affiliated Hospital of Nanjing Medical University, Nanjing, Jiangsu 210029, P.R. China

2 Department of Respiratory and Critical Care Medicine, Shanghai Pulmonary Hospital, Tongji University School of Medicine, Shanghai 200433, China muscle cells (PASMCs). This eventually results in irreversible pulmonary vascular remodeling and right ventricular (RV) failure [1]. However, the available therapeutic choices for patients with $\mathrm{PH}$ are limited and often unaffordable. Therefore, it is important to understand the complex molecular mechanisms underlying the development of $\mathrm{PH}$ and identify the potential therapeutic targets for the treatment of PH.

Dipeptidyl peptidase IV (DPP-4), also known as CD26, is a $110 \mathrm{kD}$ type II transmembrane glycoprotein firstly identified on the surface of a specific set of $\mathrm{T}$ lymphocytes 40 years ago. Now, it is known to be expressed by a variety of tissues (including lung tissue) and cells including smooth muscle cells (SMCs) and endothelial cells (ECs) [2]. DPP-4 is a serine protease belonging to the prolyl oligopeptidase family, which selectively cleaves $\mathrm{N}$-terminal dipeptides from its substrates. Moreover, a soluble form of DPP-4 is also present in the extracellular fluid such as plasma.

Recently, DPP-4 has been proposed as a diagnostic or prognostic marker for inflammatory diseases, various tumors, neuropsychiatric disorders, immune dysfunctions, and so on [3, 4]. Moreover, DPP-4 inhibitor (DPP-4i) has been reported to reduce ischemia/reperfusion injury [5], 
alleviate osteoporosis [6], and prevent diabetic-induced renal fibrosis [7]. Patients or animals with cardiovascular diseases also benefit from DPP-4i. For instance, DPP-4i could promote the left ventricular (LV) function in chronic heart failure [8], improve endothelial function, reduce atherosclerotic lesion formation [9], decrease macrophage infiltration in aortic and coronary arteries [10], enhance endothelium-dependent relaxation of mesenteric arteries [11], attenuate neointima formation after vascular injury [12], suppress proliferation of vascular SMCs [13], and eventually reduce cardiovascular remodeling and systolic pressure [14].

PH is characterized by extensive inflammatory cell infiltration, hypertrophy and hyperplasia of PASMCs, neointima formation, and terminal RV failure. We hypothesized that patients/animals with $\mathrm{PH}$ might benefit from the multiple effect of DPP-4i on the circulation system. In this study, sitagliptin (SG), a classical DPP-4i, was used to determine the effects of DPP-4 on monocrotaline (MCT)induced $\mathrm{PH}$ in rats, and the underlying mechanisms were investigated. Moreover, the therapeutic effects of SG were further validated in other PH models including bleomycin (BLM) and chronic hypoxia-induced PH.

\section{Materials and methods}

\section{Ethics statement}

All procedures in the present study were performed according to the National Institutes of Health Guide for the Care and Use of Laboratory Animals (publication no. 85-23, revised 1996) and approved by the Institutional Animal Care and Use Committee of Nanjing Medical University (NJMU/IACUC-1601196).

\section{Experimental protocol}

Forty-eight male Wistar rats $(200 \pm 10 \mathrm{~g})$ (Bikai Laboratory Animal Company, Shanghai, China) were raised under standard laboratory conditions with free access to water and food. After 1 week, 16 rats were randomly injected intraperitoneally with $0.8-\mathrm{mL}$ of saline, and the rest were injected intraperitoneally with MCT $(60 \mathrm{mg} / \mathrm{kg}$, Sigma-Aldrich, MO, USA). Of the rats receiving saline, eight were treated with SG daily $(80 \mathrm{mg} / \mathrm{kg}$, gavage, Januvia, Merck Sharp, UK), and the others were treated with $0.9 \%$ saline. Of the rats receiving MCT, eight were treated with $0.9 \%$ saline daily $(2.5 \mathrm{~mL} / \mathrm{kg}$, gavage $)$, and the rest were treated with different concentrations of SG $(20,40$, and $80 \mathrm{mg} / \mathrm{kg}$, gavage). The body weights of the rats were measured every 3 days for dose adjustment. After 28 days, the rats were sacrificed for the following examinations.
Twenty-four rats were randomly assigned into three groups. On the first day, one group received $50-\mu \mathrm{L} 0.9 \%$ saline intratracheally, when the other two groups received bleomycin hydrochloride (4 U/kg, Nippon Kayaku, Tokyo, Japan) with/without $\mathrm{SG}$ administration $(80 \mathrm{mg} / \mathrm{kg}$, gavage) in the following 4 weeks.

Moreover, another 24 rats were randomly assigned into three groups. One group was raised under normal oxygen, when the other two groups were housed in a hypoxia chamber (Biospherix Ltd., USA) for 4 weeks in which the fraction of oxygen was maintained at $10 \%$ with/without SG administration $(80 \mathrm{mg} / \mathrm{kg}$, gavage). After 28 days, the rats were sacrificed for the following examinations.

\section{Hemodynamic measurement and specimen collection}

After 28 days, the rats were anesthetized using urethane $(1.0 \mathrm{~g} / \mathrm{kg}$, intraperitoneal) and RV systolic pressure (RVSP) was measured by right-heart catheterization as described in a previous study [15]. Then, the rats were sacrificed, and heparinized blood was collected for measuring DPP-4 in plasma using the Human Dipeptidyl peptidase IV ELISA Kit (CUSABIO Life science, Wuhan, China). The lung and heart tissues were collected for histological analysis, and Western blot. Human lung paracancerous tissue for investigating DPP-4 distribution were provided by the Department of Pathology of Jiangsu Province Hospital with consent from the Bioethic Committee of the First Affiliated Hospital of Nanjing Medical University (2017-SR-198). The RV hypertrophy index (RVHI) was calculated as the weight ratio of $\mathrm{RV}$ to $(\mathrm{LV}+$ interventricular septum).

\section{Evaluation of pulmonary arterial remodeling}

A portion of the collected RV and lung tissues was immediately fixed with $4 \%$ polyformaldehyde $(\mathrm{pH} \mathrm{7.4)}$ ) for $24 \mathrm{~h}$ and embedded in paraffin. It was then sliced into $5-\mu \mathrm{m}$ sections. The sections were processed using hematoxylin and eosin (HE) staining, gomori aldehyde fuchsin (GAF) staining, picrosirius red (PSR) staining, toluidine blue (TB) staining, Masson trichrome staining (MTS), and immunohistochemical staining with primary antibody against DPP-4 (Abcam, MA, USA), smooth muscle alpha actin ( $\alpha$-SMA, Abcam), tryptase (Abcam), CD68 (Abcam), proliferating cell nuclear antigen (PCNA, Abcam), caspase-3 (Cell Signaling Technology, MA, USA), and CD31 (Abcam). All these procedures were carried out according to the protocols described in a previous study [15].

Pulmonary arterial wall thickness (PAWT) was calculated based on the results of GAF staining using the following formula: (external diameter - internal diameter)/ external diameter. The muscularization of distal vessels 
Table 1 The primer sequences of targeted RNA

\begin{tabular}{llll}
\hline Gene primer & Species & & Sequence $\left(5^{\prime}\right.$ to 3$)$ \\
\hline vimentin & Rat & Forward & GCAAAGCAGGAGTCAAACGAATA \\
& & Reverse & TAGCAGCTTCAAGGGCAAAATTC \\
fibronectin & Rat & Forward & GAGGCACAAGGTCCGAGAAGAG \\
& & Reverse & GAAACCGTGTAAGGGTCAAAGCA \\
ve-cadherin & Rat & Forward & GCCAATACTTCCGAATAACCAAAC \\
& & Reverse & TGTCATTCTCATCCAAAACTTCAA \\
vwf & Rat & Forward & CCAGACACTTGCGTTCATC \\
& & Reverse & CTACGGCTTGCACTATTCA \\
$\beta$-actin & Rat & Forward & CTGAACCCTAAGGCCAACCG \\
& & Reverse & GACCAGAGGCATACAGGGACAA \\
\hline
\end{tabular}

with diameter $30-100 \mu \mathrm{m}$ was evaluated based on the results of $\alpha$-SMA staining. Vessels exhibiting a circumference of more than $75 \%$ and positive for $\alpha$-SMA were identified as completely muscularized arteries (Fully), those exhibiting a circumference of $25-75 \%$ as partly muscularized arteries (Partly), and those exhibiting a circumference of less than $25 \%$ as nonmuscularized arteries (None). The average cross-sectional area (CSA) of 200 myocardial cells from each section was calculated using Image Pro Plus 6.0 based on HE staining results. Granulated mast cells (MCs, intact MCs with dense cytoplasm) were separated from degranulated MCs (light cytoplasm with empty spots) based on the extrusion of secretory granules using TB staining. An index of granulation (IOG) (number of granulated MCs/ number of degranulated MCs) was determined. All the graphs were observed using a Leica 2500 microscope (Leica Microsystems, Wetzlar, Germany) at different magnification.

\section{Western blot and RT-qPCR}

As described previously [16], protein extracted from lung tissues and human pulmonary arterial smooth cells (PASMCs) were separated via 10\% SDS-PAGE, electroblotted to a PVDF membrane, and incubated with primary antibodies against DPP-4 (Abcam), $\alpha$-SMA (Abcam), von Willebrand factor (vWF, Proteintech, Rosemont, IL), caspase3 (CST), phosphatase, and tensin homolog deleted on chromosome ten (PTEN, CST), extracellular regulated protein kinases 1/2 (ERK1/2, CST), phosphorylated ERK1/ 2 (p-ERK1/2, CST), protein kinase B (AKT, CST), phosphorylated AKT (p-AKT, CST), c-Jun N-terminal kinase (JNK, CST), phosphorylated JNK (p-JNK, CST), mitogenactivated protein kinase (MAPK) p38 (CST), phosphorylated p38 (CST), and $\beta$-actin (Proteintech). Over $16 \mathrm{~h}$ later, PVDF membrane was incubated with HRP-conjugated second antibody and chemiluminescence (ECL, CST) were employed to label protein band. Band intensities were analyzed densitometrically using Image Lab 4.1 software.

RNA derived from lung tissues and human PASMCs was extracted using Trizol reagent (Gibco BRL, Grand Island, NY). Reverse transcription was conducted with $2000 \mathrm{ng}$ of total RNA with SYBR Premix Ex Taq ${ }^{\mathrm{TM}}$ kit (TaKaRa Bio Inc., Dalian, China). Quantitative RCR was performed using an ABI Prism 7500 FAST apparatus (Applied Biosystems, Foster City, CA, USA). $\beta$-actin was selected as housekeeping gene. PCR primers are listed in Table 1.

\section{Cell culture and proliferation assay}

Human PASMCs were purchased from ScienCell (CA, USA) and maintained in a SMC medium (SMCM, ScienCell) containing $10 \mu \mathrm{g} / \mathrm{mL}$ streptomycin, $10 \mathrm{U} / \mathrm{mL}$ penicillin, SMC growth supplement, and $2 \%$ heat-inactivated fetal bovine serum (ScienCell). The cells were incubated in a $5 \% \mathrm{CO}_{2}$ humidified incubator at $37^{\circ} \mathrm{C}$.

The human PASMCs (passages 7-9) were quiesced for $48 \mathrm{~h}$ in SMCM without growth supplement and serum before performing the proliferation-related experiment. Then, SG at a given concentration was added to incubate human PASMCs for $24 \mathrm{~h}$. Recombinant human plateletderived growth factor-BB (PDGF-BB, $20 \mathrm{ng} / \mathrm{mL}$, PeproTech, NJ, USA) was added to stimulate human PASMCs for some more time. Twenty-four hours after human PASMCs were challenged with PDGF-BB, cells were collected for the following measurements. Cell counting kit-8 assay was used to examine the cell viability of human PASMCs at $450 \mathrm{~nm}$ using a microplate reader (Thermo Scientific, CA, USA). The EdU (5-ethynyl-2'-deoxyuridine) incorporation assay was performed to determine the proliferation of human PASMCs under a Leica 2500 microscope. Propidium iodide (PI) staining was performed to investigate the cell cycle of human PASMCs, and Annexin $\mathrm{V}+\mathrm{PI}$ staining was used to detect the apoptosis of human PASMCs using a FACScan flow cytometer (Beckman Coulter, CA, USA). 
A
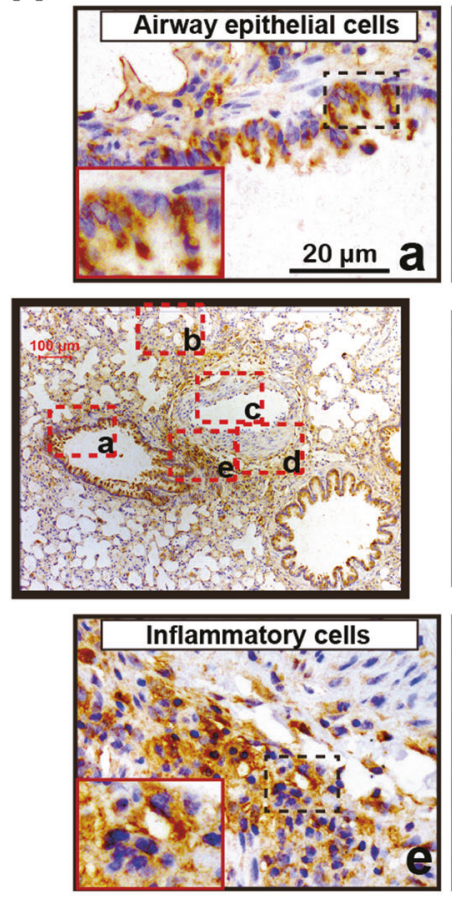

C

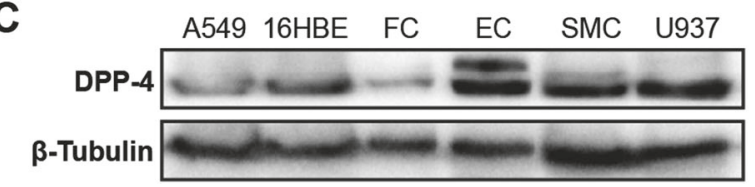

B
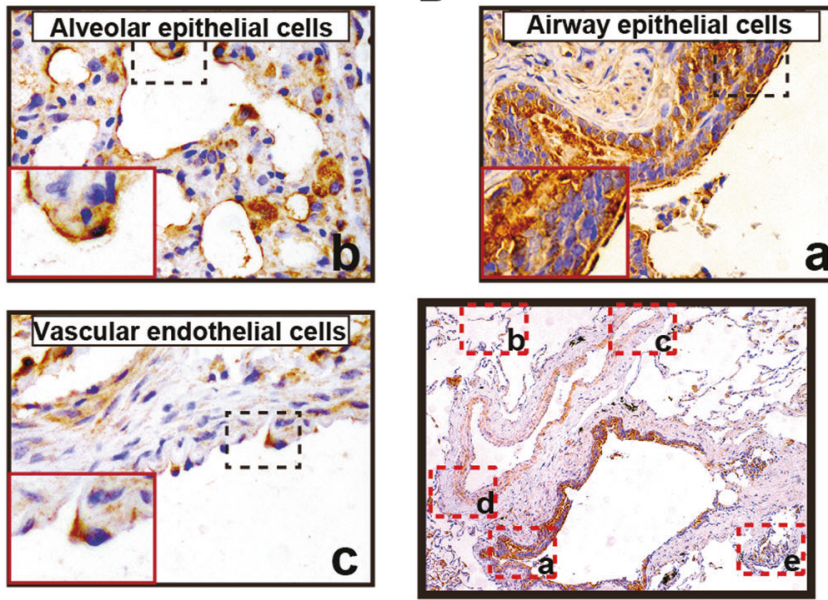

a
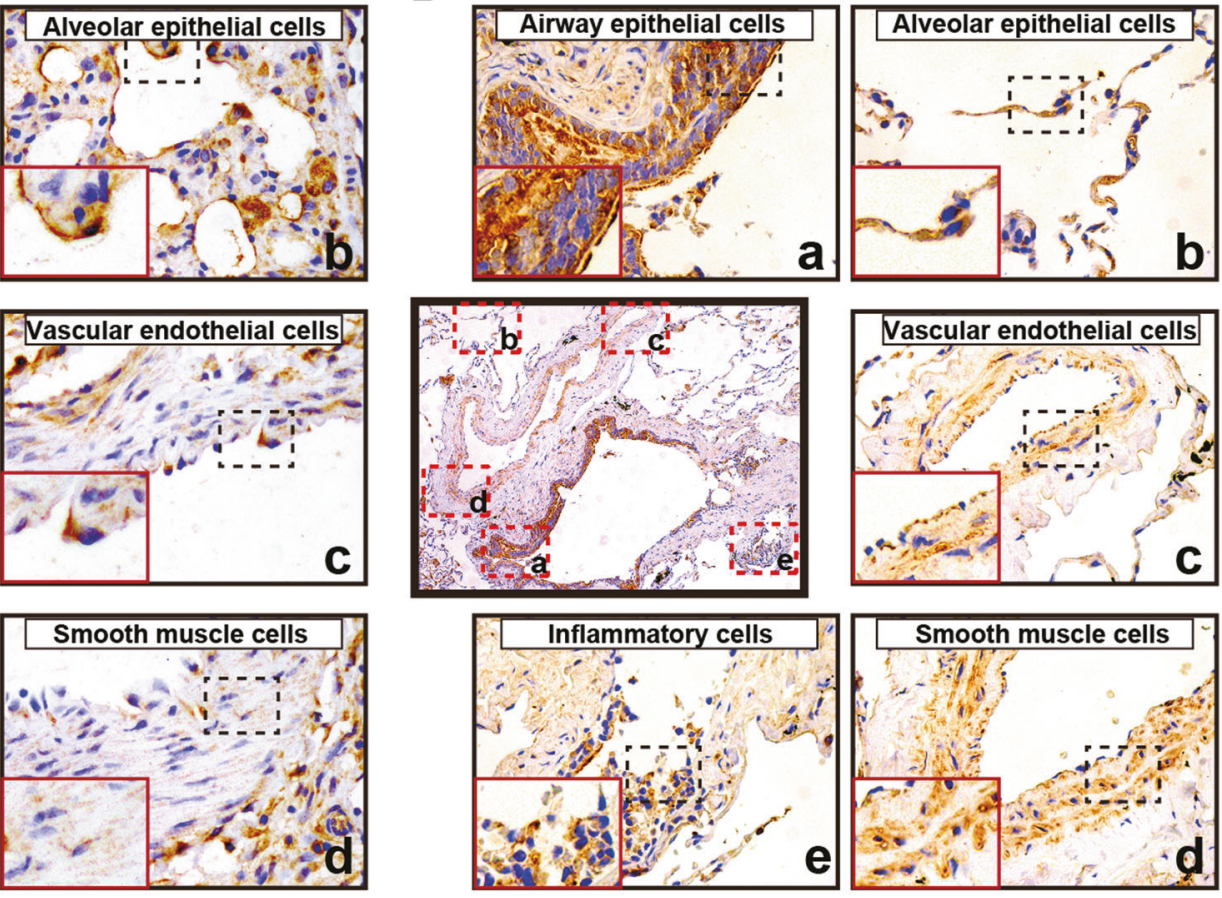

Fig. 1 Distribution of Dipeptidyl peptidase IV (DPP-4) in lung tissues from rat (a) and human (b). c Total protein extracted from A549 cells (original from alveolar epithelial cells), 16HBE cells (original from airway epithelial cells), human lung fibroblasts (FC), human

\section{Knockout using CRISPR}

sgRNA-plasmid targeting human PTEN (sgPten) was purchased from Santa Cruz Biotechnology (sc-400103). Control CRISPR/Cas9 Plasmid (sc-418922) was used as a negative control (sgGFP). The sequences of sgRNA targeting human PTEN are as follows:

sgPTEN_\#1F: ACCGCCAAATTTAATTGCAG

sgPTEN_\#2F: CCTACCTCTGCAATTAAATT

sgPTEN_\#3F: TTATCCAAACATTATTGCTA

PTEN CRISPR/Cas9 KO plasmids along with HDR plasmids were transiently transfected into cells using lipofectamine 2000. Seventy hours later, successfully transfected cells were purified using puromycin selection. Four days later, cells were collected for Western blot analysis or other experiment.

\section{Cell migration assay}

For the Transwell migration chamber assay, PDGF-BB with/without SG were added to the lower chamber when pulmonary arterial endothelial cells (EC), human pulmonary arterial smooth muscle cells (SMC), and U937 (mononuclear cells) was detected by Western blot with primary antibody against DPP-4

human PASMCs $\left(5 \times 10^{4}\right.$ cells in $100 \mu \mathrm{L}$ serum-free medium) were placed in the upper chamber. Twelve hours later, non-migrated cells were wiped out gently from the top side. Human PASMCs migrating onto the lower side of the membrane were fixed with $4 \%$ formaldehyde, stained with 5\% Crystal violet, and counted under Leica 2500 microscope. For wound healing assay, human PASMCs were cultured till $100 \%$ confluence, serum deprivation for $48 \mathrm{~h}$, and then wounded with a sterile $100 \mu \mathrm{L}$ pipette tip. After incubating with PDGF-BB with/without SG for $12 \mathrm{~h}$, migrated human PASMCs were calculated under a phase contrast microscope (Nikon, Tokyo, Japan).

\section{Statistical analysis}

All data are presented as mean \pm s.e.m. Differences between the two groups were analyzed using unpaired $t$-test. Wherever appropriate, comparisons among groups were performed using one-way ANOVA followed by least significant difference post (LSD) post hoc test. A $P$ value $<$ 0.05 was considered statistically significant. 
A

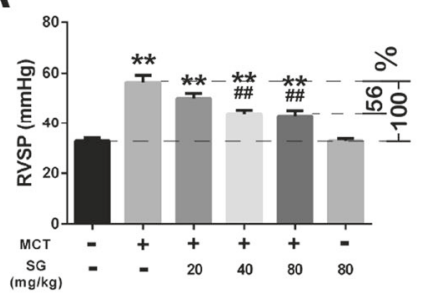

B

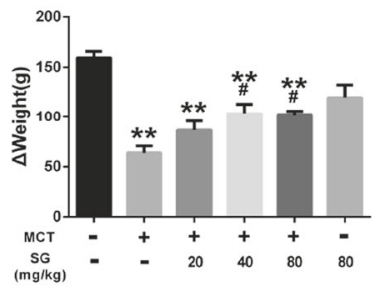

C

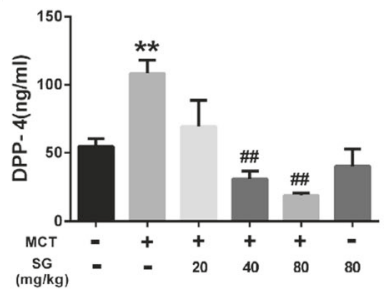

D
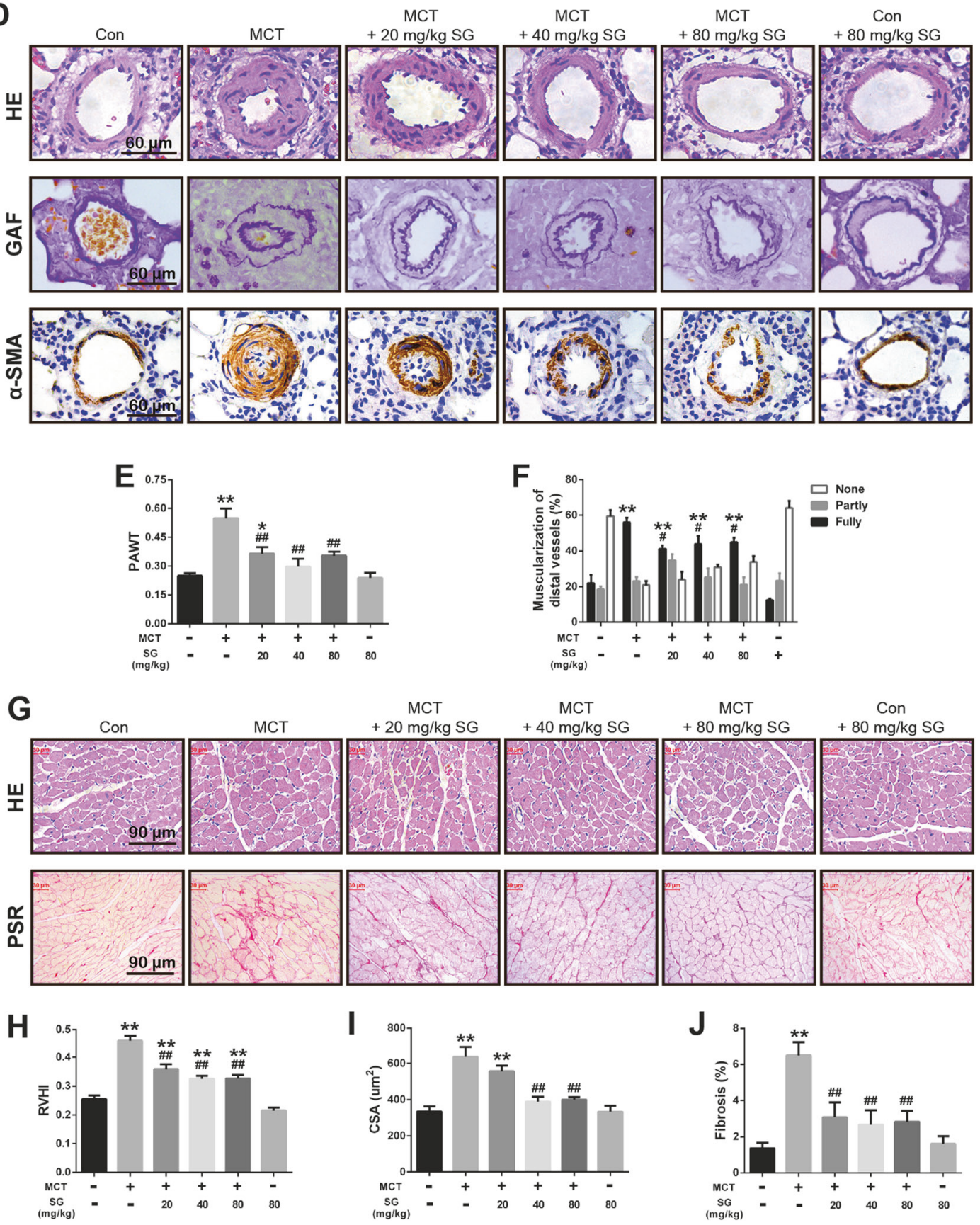
Fig. 2 Sitagliptin (SG) attenuated right ventricular systolic pressure (RVSP), the hypertrophy of pulmonary arterial medial layer, and right ventricle (RV) remodeling in rats with monocrotaline (MCT)-induced pulmonary hypertension (PH). a RVSP. b Weight gain of rats during the 4 weeks ( $\Delta$ Weight). c Concentration of DPP-4 in serum. d Representative photomicrographs from lung tissues with hematoxylin and eosin staining (HE), gomori aldehyde fuchsin staining (GAF), and immunostaining with smooth muscle alpha actin ( $\alpha$-SMA) antibody. $\mathrm{e}$ Pulmonary arterial wall thickness (PAWT) was calculated according to GAF. f Percent of completely muscularized arteries (Fully), partly muscularized arteries (Partly), and non muscularized arteries (None) were determined by $\alpha$-SMA staining. g Representative photomicrographs from RV with HE and picrosirius red staining (PSR). $\mathbf{h}$ RV hypertrophy index (RVHI). i Mean cross section area (CSA) of cardiomyocytes was evaluated based on the results of HE. $\mathbf{j}$ Fibrosis of $\mathrm{RV}$ was calculated using PSR. Data are presented as the mean \pm s.e.m. $(n=6-8)$. $* P<0.05$ compared to control; $* * P<0.01$ compared to control; ${ }^{\#} P<0.05$ compared to MCT; ${ }^{\# \#} P<0.01$ compared to MCT

\section{Results}

\section{Distribution of DPP-4 in lung tissue}

Immunohistochemistry study showed that DPP-4 was distributed in airway epithelial cells, alveolar epithelial cells, vascular ECs, SMCs, and inflammatory cells in the lung tissues from PH rat (Fig. 1a) as well as lung tissues from human being with lung cancer (Fig. 1b).

Total protein extracted from cultured A549 cells (original from alveolar epithelial cells), 16HBE cells (original from airway epithelial cells), human lung fibroblasts, human pulmonary arterial ECs, human PASMCs, and U937 (mononuclear cells) were used to validate the expression patterns of DPP-4 in lung tissues. As shown in Figure 1c, Western blot analysis confirmed that DPP-4 was expressed in all of these cells.

\section{SG attenuated RVSP, hypertrophy of pulmonary arterial medial layer, and $\mathrm{RV}$ remodeling in rats with MCT-induced PH}

After 28 days, the RVSP in rats injected with MCT was significantly higher than that in rats injected with saline, but RVSP decreased with SG treatment in a dose-dependent manner (Fig. 2a). Additionally, the reduction of weight gain ( $\Delta$ weight) in rats challenged with MCT was alleviated by SG in a dose-dependent manner (Fig. 2b). In addition, SG inhibited MCT-induced increase of DPP-4 in serum (Fig. 2c). HE and GAF staining were employed to determine hypertrophy of pulmonary arterial medial layer (Fig. 2d). As indicated in Figure 2e, PAWT in MCT-treated rats increased significantly compared to those in salinetreated rats, which was attenuated by SG. Immunohistochemical staining against $\alpha$-SMA was performed to evaluate muscularization in small pulmonary vessels (outer diameter of 30-100 $\mu \mathrm{m}$ ) (Fig. 2d). The ratio of completely muscularized vessels (Fully) only accounted for $16.96 \pm$ $15.73 \%$ in saline-treated rats, while it increased to $55.89 \%$ $\pm 7.03 \%$ in MCT-treated rats. However, it was significantly decreased by SG at different doses (Fig. 2f). RVHI, CSA of $\mathrm{RV}$ cardiomyocytes, and RV fibrosis were calculated to evaluate the effect of $\mathrm{SG}$ on $\mathrm{RV}$ remodeling. Representative pathological changes with HE and PSR staining are shown in Figure 2g. Statistical analysis indicate that SG effectively alleviated MCT-induced increments in RVHI (Fig. 2h), CSA (Fig. 2i), and RV fibrosis (Fig. 2j). Together, these results demonstrate that SG could prevent MCT-induced pulmonary hypertension, pulmonary arterial remodeling, and right ventricle dysfunction.

\section{SG alleviated the remodeling of pulmonary arterial adventitia in rats with MCT-induced PH}

Remodeling of pulmonary arterial adventitia in $\mathrm{PH}$ was characterized by extensive fibrosis and inflammatory cell infiltration. Representative images with MTS staining are presented in Figure 3a. Extensive pulmonary arterial adventitial fibrosis in MCT-treated rats was reduced by SG administration (Fig. 3b) at doses 40 and $80 \mathrm{mg} / \mathrm{kg}$. Perivascular inflammatory cell infiltration, including MCs and macrophages, was labeled with primary antibodies against tryptase and CD68 respectively (Fig. 3a). MCT induced significant accumulation of MCs and macrophages around pulmonary arteries, which were inhibited by SG (Fig. 3c, e). Moreover, degranulated MCs and granulated MCs were identified by TB staining (Fig. 3a). As shown in Figure 3d, index of granulation (IOG) decreased remarkably in MCTtreated rats, whereas it was partially reversed by SG.

\section{SG attenuated endothelial-mesenchymal transition (EndMT) in pulmonary arterial intima in rats with MCT-induced PH}

EndMT in pulmonary arterial intima is also involved in the vascular remodeling of $\mathrm{PH}$. Using immunofluorescence staining against mesenchymal marker $\alpha$-SMA (green) and endothelial marker CD31 (red), cells undergoing EndMT (double-labeled, yellow) were scattered in the pulmonary arteries of MCT-treated rats, while few double-labeled cells were observed in control rats or SG-treated rats (Fig. 4a). The expressions of characteristic markers of EndMT in pulmonary vessels were further determined by Western blot and RT-qPCR. As shown in Figure 4b, c, we found a downregulation of endothelial specific marker vWF and VEcadherin, as well as an up-regulation of mesenchymal marker $\alpha$-SMA, vimentin and fibronectin in MCT-treated rats. However, these changes were partially reversed by SG treatment. 


\section{SG prevented bleomycin- and hypoxia-induced PH}

The therapeutic effects of SG were further investigated in bleomycin- and chronic hypoxia-induced PH. Bleomycin, which could induce pulmonary fibrosis, was used to induce $\mathrm{PH}$ in rats. As expected, a remarkable increase in RVSP, PAWT, RVHI, and CSA of RV cardiomyocytes was observed in rats challenged with bleomycin hydrochloride (Fig. 5a-d). At the concentration of $80 \mathrm{mg} / \mathrm{kg}$, SG effectively alleviated these alternations. In addition, chronic hypoxia for 28 days resulted in significantly increased RVSP, PAWT, RVHI, and CSA of RV cardiomyocytes (Fig. 5e-h), which were also suppressed by the treatment of SG $(80 \mathrm{mg} / \mathrm{kg})$.

\section{SG inhibited PDGF-BB-induced proliferation of human PASMCs via inhibiting Akt/MAPK signaling by PTEN up-regulation}

Hyper-proliferated PASMCs result in hypertrophy of pulmonary arteries. After serum deprivation for $48 \mathrm{~h}$, human PASMCs were pre-treated with SG at the indicated concentrations $(2.5,5,10,20$, and $40 \mu \mathrm{M})$ for $24 \mathrm{~h}$ with/without PDGF-BB stimuli $(20 \mathrm{ng} / \mathrm{mL})$ for another $24 \mathrm{~h}$. As shown in Figure 6a, PDGF-BB challenge enhanced cell viability of human PASMCs, which was attenuated by SG in a dosedependent manner. However, SG alone had no remarkable effect on the cell viability (Fig. 6b). EdU staining was further applied for proliferation assay. As indicated in
A
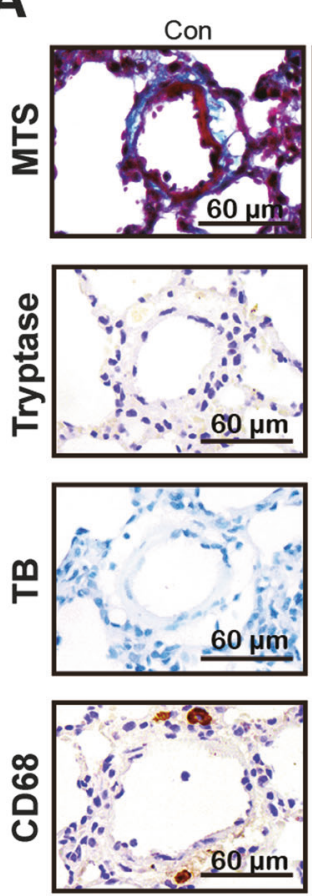

B

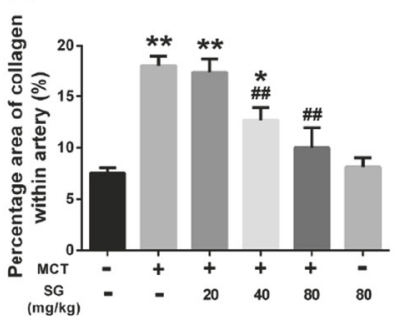

C

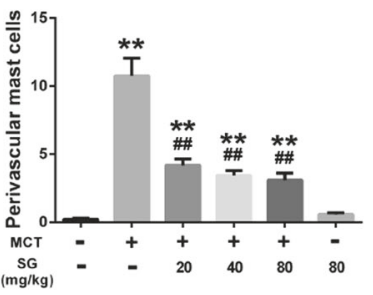

Fig. 3 SG alleviated the remodeling of pulmonary arterial adventitia in rats with MCT-induced PH. a Representative photomicrographs with masson trichrome staining (MTS), toluidine blue staining (TB), and immunostaining against tryptase and CD68. b Pulmonary vascular fibrosis was calculated based on the results of MTS. c Infiltration of mast cells was evaluated using immunostaining against tryptase. d
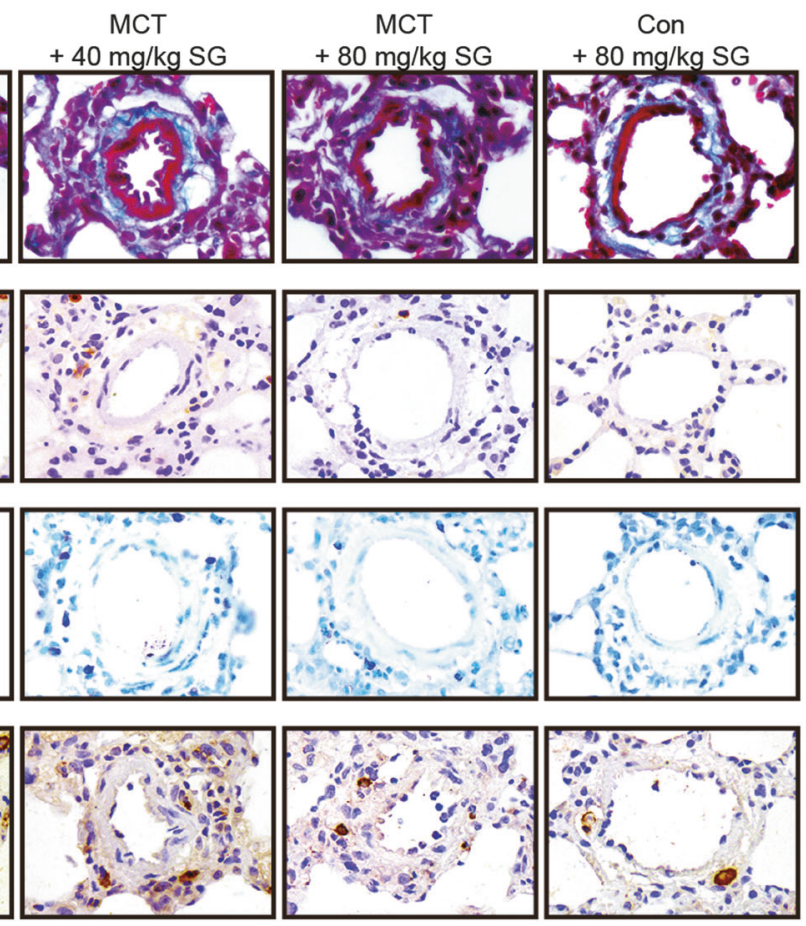

E
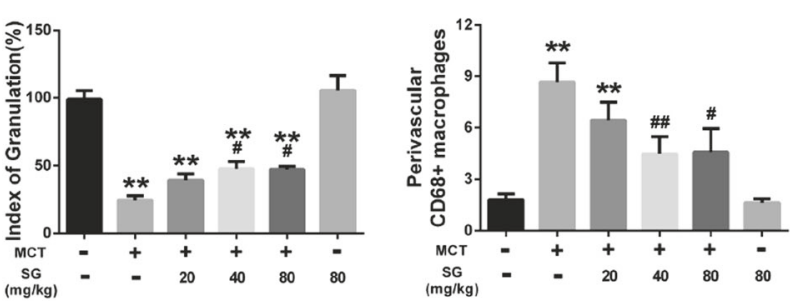

Index of granulation in mast cells was determined by TB staining. $\mathbf{e}$ Evaluation of macrophages infiltration was performed by immunostaining against CD68. Data are presented as the mean \pm s.e.m. $(n=$ 4-6). $* P<0.05$ compared to control; $* * P<0.01$ compared to control; ${ }^{\#} P<0.05$ compared to MCT; ${ }^{\# \#} P<0.01$ compared to MCT 


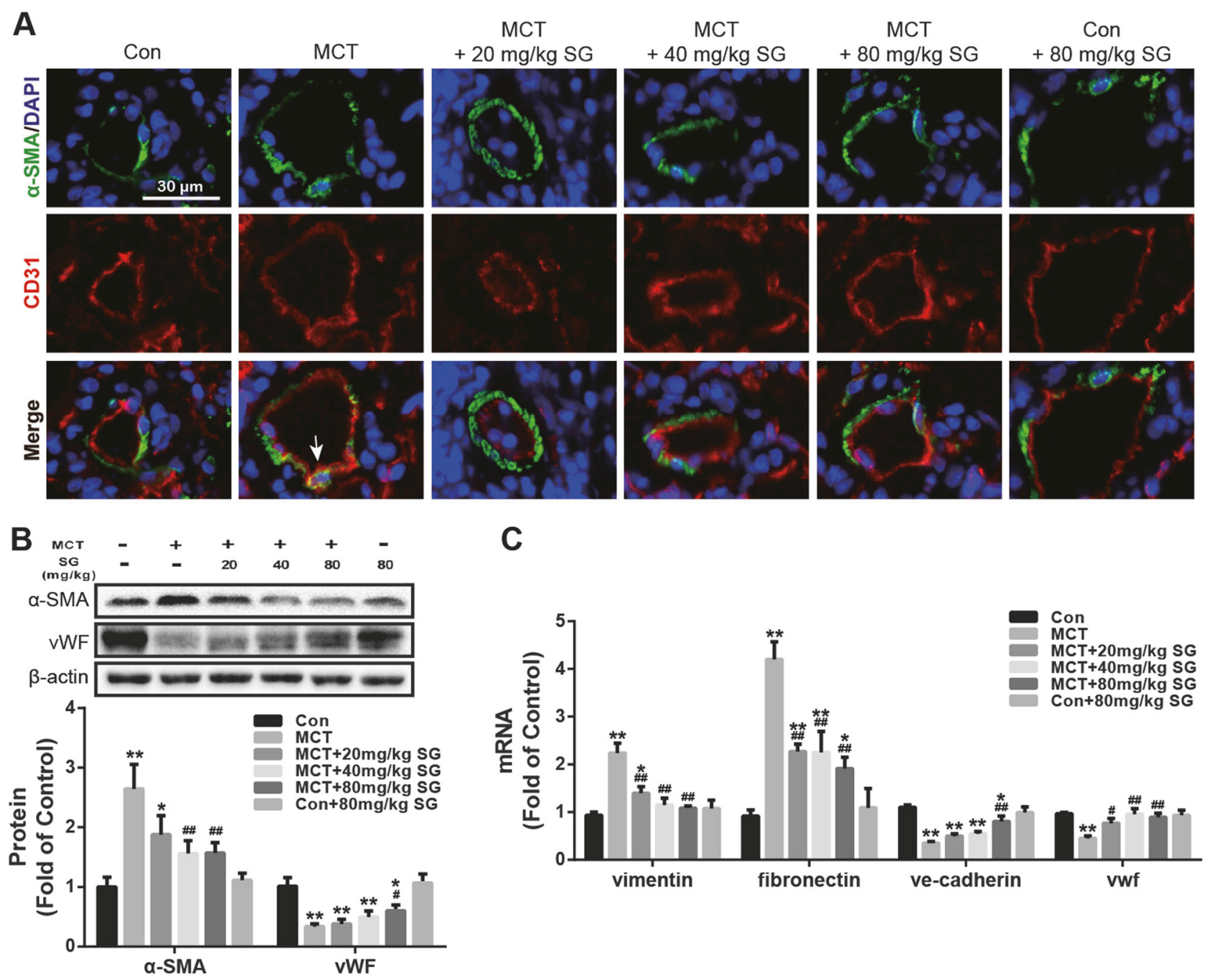

Fig. 4 SG attenuated endothelial-mesenchymal transition (EndMT) in rats with MCT-induced $\mathrm{PH}$. a Representative photomicrographs with immunofluorescence staining against $\alpha$-SMA (green) and CD31 (red), cells exhibiting yellow (white arrow) is undergoing EndMT. b Semiquantitative analysis of $\alpha$-SMA and von Willebrand factor (vWF) with

Western blot. c Quantitative analysis of vimentin, fibronectin, vecadherin, and vwf using RT-qPCR. Data are presented as the mean $\pm \mathrm{s}$. e.m. $(n=4-6)$. $* P<0.05$ compared to control; $* * P<0.01$ compared to control; ${ }^{\#} P<0.05$ compared to MCT; ${ }^{\# \#} P<0.01$ compared to MCT

Figure 6c, few EdU-positive cells were found in human PASMCs without PDGF-BB stimuli. After $20 \mathrm{ng} / \mathrm{mL}$ PDGF-BB challenge for $24 \mathrm{~h}$, more than $15 \%$ cells were EdU positive. Nevertheless, SG $(40 \mu \mathrm{M})$ significantly reduced the percent of EdU-positive human PASMCs. Cell cycle assay by flow cytometry showed that $40 \mu \mathrm{M}$ SG significantly inhibited PDGF-BB-induced increase in the percentage of S phase (Fig. 6d).

PTEN has been recognized as an upstream inhibitor of Akt in regulating cell proliferation and apoptosis. SG significantly increased the expression of PTEN, while PDGFBB showed an inhibitory effect (Fig. 6e). In contrast, the phosphorylation of Akt was significantly induced by the treatment of PDGF-BB, which was inhibited by the pretreatment of SG (Fig. 6f). MAPK signaling pathways,

including p38, ERK1/2, and JNK, also play important roles in PDGF-BB-induced proliferation of human PASMCs. Our results indicated that PDGF-BB significantly resulted in phosphorylation of p38, ERK1/2, and JNK. Pretreated with SG inhibited PDGF-BB-induced phosphorylation of p38 and ERK1/2, but had no effect on JNK (Fig. 6f).

To further confirm whether the anti-proliferative effect of SG in human PASMCs was dependent on PTEN signaling, PTEN expression in human PASMCs was knocked out using PTEN CRISPR/Cas9 KO Plasmid (Fig. 6g). The results obtained from EdU staining showed that the antiproliferative effect of SG on PDGF-BB-induced proliferation of human PASMCs was abolished by PTEN knockout (Fig. 6h). 
A

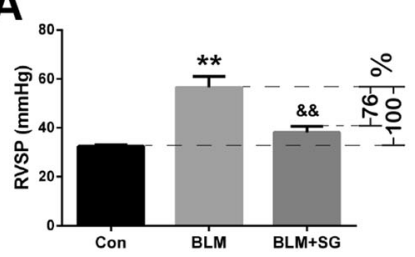

C

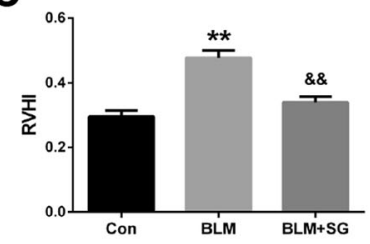

E
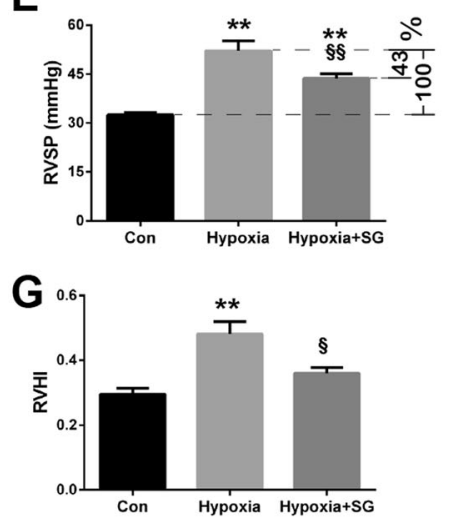

Fig. 5 SG prevented bleomycin (BLM)- and hypoxia-induced PH. a RVSP. b Representative photomicrographs and graphic analysis of HE staining in lung tissues. c RVHI. d Representative photomicrographs and graphic analysis of HE staining in RV tissues. e RVSP. f Representative photomicrographs and graphic analysis of HE staining in lung tissues. g RVHI. h Representative photomicrographs and graphic

\section{SG reduced PDGF-BB-induced migration of human PASMCs}

Increased migration of PASMCs is another mechanism for the muscularization of small pulmonary vessels. In transwell migration chamber assay, SG remarkably attenuated PDGF-BB-induced migration of human PASMCs from the top side of a chamber to the lower side (Fig. 7a). The results from wound healing experiment (Fig. 7b) were consistent with the results from transwell migration chamber assay.

However, incubation human PASMCs with PDGF-BB had no effect on cell apoptosis, while pretreatment with SG significantly induced apoptosis (Fig. 7c).

\section{Dicussion}

$\mathrm{PH}$ is a chronic cardiopulmonary disorder arising from a variety of genetic and pathogenic causes characterized by pulmonary vascular injury and remodeling that leads to elevated pulmonary vascular resistance, right heart failure,
BLM
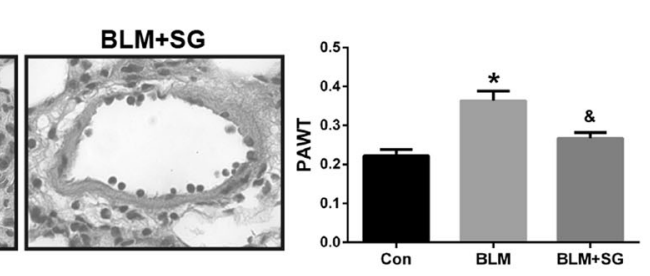

BLM+SG
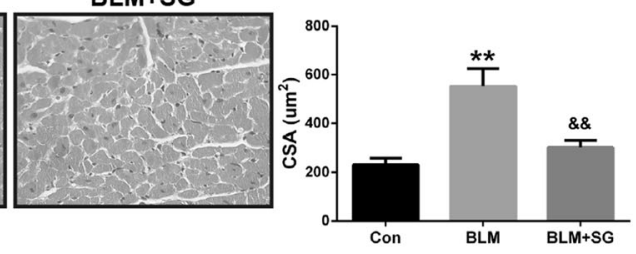

Hypoxia

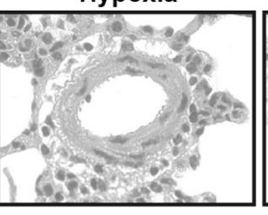

Hypoxia
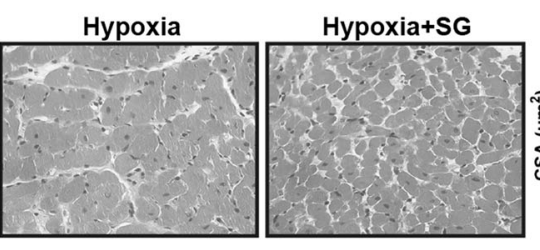

Hypoxia+SG
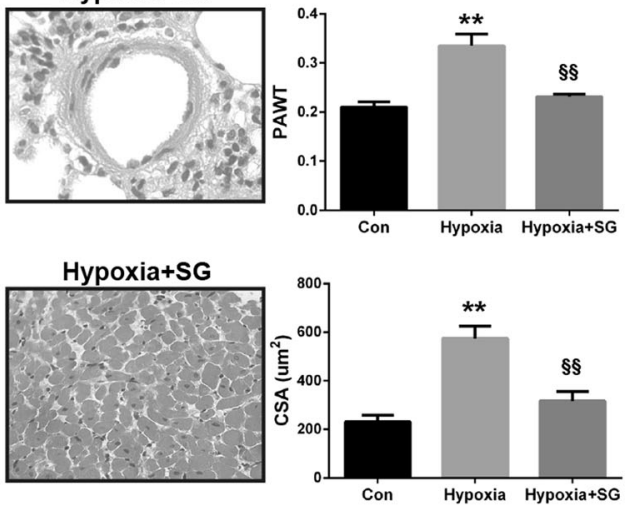

analysis of HE staining in RV tissues. Data are presented as the mean \pm s.e.m. $(n=6) . * P<0.05$ compared to control; $* * P<0.01$ compared to control; ${ }^{\&} P<0.05$ compared to $\mathrm{BLM}$; ${ }^{\& \&} P<0.01$ compared to BLM; ${ }^{\S} P<0.05$ compared to Hypoxia; ${ }^{\S \S} P<0.01$ compared to Hypoxia

and ultimately death. Pulmonary vascular remodeling is a hallmark of structural alteration in $\mathrm{PH}$, which is due to abnormal proliferation and resistance to apoptosis of ECs and PASMCs in the vascular wall, pulmonary adventitial fibrosis, and inflammatory cells infiltration. In the present study, we demonstrated that DPP-4i alleviated pulmonary arterial remodeling, RVSP, and RV remodeling in rats with MCT-induced PH, as well as bleomycin- and hypoxiainduced $\mathrm{PH}$ in rats. In vitro study indicates that DPP-4i prevent pulmonary artery remodeling by inhibiting proliferation and migration, as well as inducing apoptosis of PASMCs. Further study suggests that inhibition PTEN-AktMAPK signaling pathway contribute to the therapeutic effects of DPP4i.

DPP-4 is a glycoprotein peptidase widely expressed in different organs exhibiting complex biological roles, including enzymatic activity, intercellular interaction, and cell membrane associated activation of intracellular signal pathways [3]. The extracellular domain of DPP-4 can be cleaved from its membrane-anchored form and circulates in 

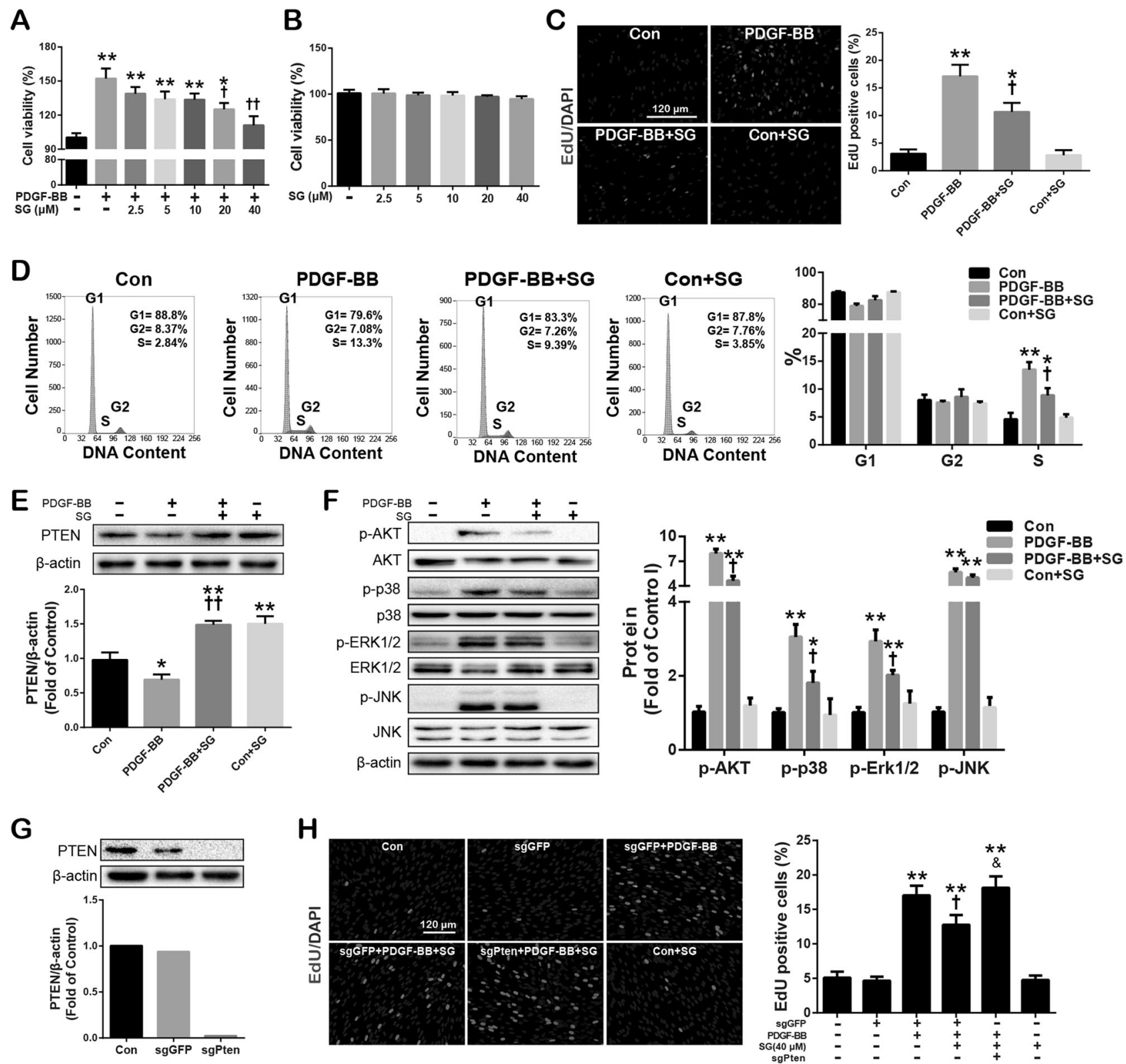

Fig. 6 SG $(20 \mu \mathrm{M})$ inhibited PDGF-BB-induced proliferation of human pulmonary arterial smooth muscle cells (PASMCs) via inhibiting Akt/MAPK signaling by phosphatase and tensin homolog deleted on chromosome ten (PTEN) upregulation. After serum deprivation for $48 \mathrm{~h}$, human PASMCs were treated with $\mathrm{SG}$ at the indicated concentrations for $24 \mathrm{~h}$ with/without PDGF-BB $(20 \mathrm{ng} / \mathrm{ml})$ stimuli for another $24 \mathrm{~h}$. a,b Cell viability detected by cell counting kit- 8 assay (CCK-8). c Proliferated cells were labeled by EdU (red) when cell nucleus labeled by DAPI (blue). d Analysis of cell cycle using a FACScan flow cytometer. e Semiquantitative analysis of PTEN with Western blot. f After treatment with PDGF-BB for $30 \mathrm{~min}$ in the

plasma, where it retains full enzymatic activity [2]. By immunohistochemistry study, we found that membranebound DPP-4 was distributed in airway epithelial cells, PASMCs, pulmonary vascular ECs, and perivascular infiltrated inflammatory cells in both rat lung tissues and human lung tissues. Of note, the abundance of DPP-4 in pulmonary presence or absence of SG $(20 \mu \mathrm{M})$, cells were harvested for semiquantitative analysis of p-p38, p-ERK1/2, p-JNK, and p-AKT with Western blot. $\mathrm{g}$ Expression of PTEN after cells was transfected with sgPten or sgGFP for $72 \mathrm{~h}$. $\mathbf{h}$ EdU incorporation assay in the presence or absence of SG $(20 \mu \mathrm{M})$ or sgPten. Data are presented as the mean \pm s.e.m. $(n=3-4)$. $* P<0.05$ compared to control; $* * P<0.01$ compared to control; ${ }^{\dagger} P<0.05$ compared to PDGF-BB; ${ }^{\dagger} P<0.01$ compared to PDGF-BB; ${ }^{\&} P<0.05$ compared to PDGF-BB + SG. AKT protein kinase $\mathrm{B}$, MAPK mitogen-activated protein kinase, ERK1/2 extracellular regulated protein kinases $1 / 2$, JNK c-Jun N-terminal kinase

vascular smooth muscle cells (PVSMCs) of rat is different from that of human beings as presented in Figure 1. We considered that it might be attributed to the species difference (samples from rat vs. human) or the disease difference (sample from MCT-induced PH rat vs. adjacent normal tissue of lung cancer). Moreover, MCT challenge induced a 
A

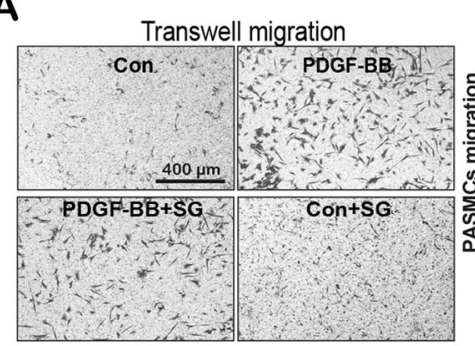

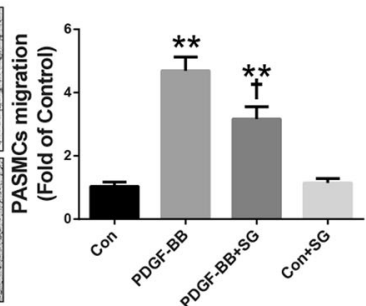

B

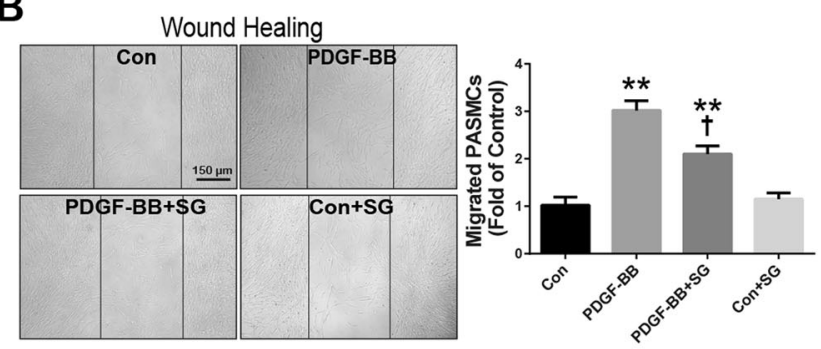

C

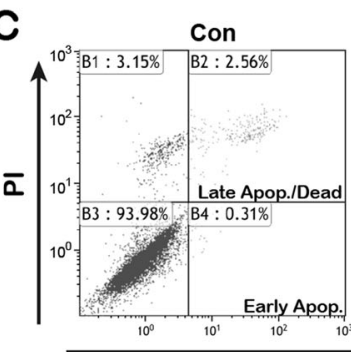

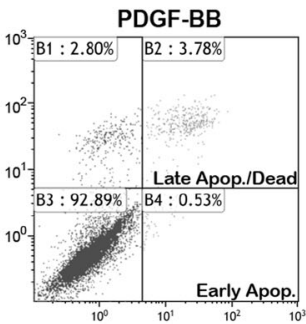

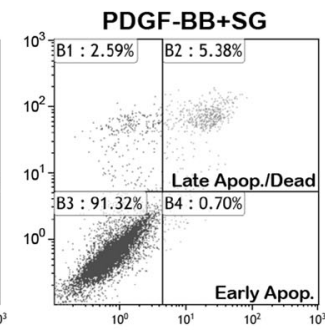

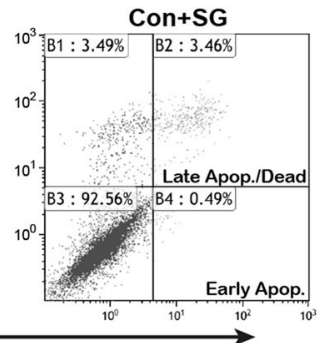

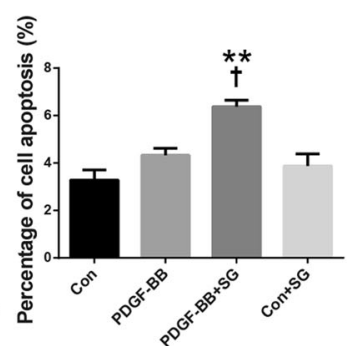

Annexin-FITC

Fig. 7 SG reduced PDGF-BB-induced migration of human PASMCs, and induced apoptosis of human PASMCs. a,b Representative photomicrographs with transwell migration chamber assay and wound healing assay after incubation with PDGF-BB $(20 \mathrm{ng} / \mathrm{ml})$ for $12 \mathrm{~h}$. c With annexin V combining PI staining, apoptosis of human PASMCs was detected using a FACScan flow cytometer. Data are presented as the mean \pm s.e.m. $(n=3-4)$. $* P<0.05$ compared to control; $* * P<$ 0.01 compared to control; ${ }^{\dagger} P<0.05$ compared to PDGF-BB; ${ }^{\dagger \dagger} P<$ 0.01 compared to PDGF-BB

animal models of heart or kidney diseases [17, 22-24], which might be attributed to the antifibrotic miRNA induction, DPP4-integrin- $\beta 1$ interaction suppression, and TGF- $\beta$ signal transduction inhibition [25]. In addition, interaction of DPP-4 and integrin $\beta 1$ facilitated renal fibrosis by enhancing TGF- $\beta$ receptor-mediated EndMT [26]. Accordingly, DPP-4i showed anti-fibrotic effect by preventing EndMT in diabetic nephropathy [27]. In the current study, EndMT was found in the intima of small pulmonary arteries in rats with MCT-induced $\mathrm{PH}$, while inhibition of DPP-4 by SG effectively prevented MCTinduced EndMT. It should be noted that EndMT is also implicated in initiation and progression of $\mathrm{PH}$ [28]. Together, these results suggest that the beneficial effect of DPP$4 \mathrm{i}$ on pulmonary vessel remodeling in $\mathrm{PH}$ could be achieved by different ways including: (1) protecting endothelium against EndMT and (2) preventing adventitia fibrosis and inflammatory cell infiltration.

Our in vivo study confirmed that inhibiting DPP-4 by SG alleviated MCT-induced PH. The beneficial effects of SG on $\mathrm{PH}$ were further validated in bleomycin and chronic hypoxia-induced $\mathrm{PH}$. Thus, the therapeutic effects of SG may not depend on the etiology of pulmonary hypertension. However, SG at $80 \mathrm{mg} / \mathrm{kg}$ could reduce $56 \%$ of the increase in right ventricular systolic pressure (RVSP) in MCT-PH rats, $76 \%$ in bleomycin (BLM)-PH rats, and $43 \%$ in hypoxia-PH. Thus, the effect of SG is substantial in BLM$\mathrm{PH}$, and modest in MCT- and hypoxia-PH. As reported, DPP-4 gene presented a significant decrease in patients with fibrotic effects of DDP-4i have been confirmed in different 
pulmonary fibrosis-related $\mathrm{PH}$ and a minor decrease in patients with idiopathic pulmonary arterial hypertension (iPAH) without significance [29]. We speculate that the different expression of DPP-4 in different types of $\mathrm{PH}$ might contribute to the varied effect of SG. Further work will be carried out to evaluate the correlation between DPP-4 level in lung tissues and therapeutic potency of DPP4i.

Hypertrophy and hyperplasia of PASMC-mediated vascular medial remodeling is the prominent characteristic features of PH [30]. Our in vivo study indicates that MCTinduced hypertrophy of pulmonary vascular medial layer in rats. Further PCNA and Caspase-3 immunohistochemical studies (see supplemental material) suggest the medial hypertrophy might be attributed to the hyperproliferation and apoptosis-resistance of PAMSCs. However, inhibition of DPP-4 by SG blocked MCT-induced proliferation and reversed apoptosis-resistance. These results indicate that DPP-4 regulates the function of PAMSCs during the development of PH. Therefore, cultured human PASMCs were used for further exploring the underlying mechanism.

PDGF-BB is a potent mitogen implicated in the abnormal proliferation and migration of PAMSCs, and has been proposed as a key mediator in the progression of $\mathrm{PH}$. Our in vitro cell proliferation and cell cycle studies showed that DPP-4i suppressed PDGF-BB-induced proliferation of human PASMCs by inhibiting DNA synthesis. Previous studies reported that soluble DPP-4 itself could augment proliferation of human coronary artery smooth muscle cells via ERK1/2 signaling pathway, and this effect was blocked by DPP-4i as well as ERK1/2 inhibitor [31]. However, our present study showed that, without soluble DPP4, DPP-4i SG repressed PDGF-BB-challenged proliferation of human PASMCs. This finding is consistent with the previous reports that DPP-4i inhibited PDGF-BB-mediated proliferation in activated hepatic stellate cells [32] and aortic smooth muscle cells [33], and implies membrane-anchored DPP-4 is involved in the pro-proliferative effects of PDGFBB. Likewise, DPP-4i also suppressed PDGF-BB-induced migration of human PASMCs. There is now mounting evidence that PI3K-Akt and MAPK signaling pathways play pivotal roles in PDGF-BB-induced proliferation and migration of PASMCs $[34,35]$. In the present study, inhibition of DPP-4 by SG suppressed PDGF-BB stimulated phosphorylation of AKT, p-38, and ERK1/2, but not JNK. Notably, we found that SG reversed PDGF-BB-induced down-regulation of PTEN. PTEN is considered to be a tumor suppressor gene as well as a multifunctional lipid phosphatase. During the past decade, PTEN has been demonstrated to play a critical role in the development of PH. In human patients, a link between PH and PTEN has been reported in a patient diagnosed with a mutation in PTEN gene [36]. In experimental animal models, significant downregulation of PTEN was found in PASMCs from hypoxia-induced $\mathrm{PH}$, and PTEN knockout in PASMCs promoted an irreversible progression of $\mathrm{PH}$ [37]. In our present study, the inhibitory effect of DPP-4i on PDGF-BBinduced proliferation of human PASMCs was abolished by PTEN knockout suggesting PTEN might be an important mediator for the action of DPP-4i. It is well documented that PTEN is a main negative regulator of the PI3K-Akt pathway [38]. Moreover, PTEN modulates the activation of MAKP signaling pathways by dephosphorylating components of the MAPK cascade directly [39], or by the crosstalk between PI3K-AKT and MAPKs indirectly [40, 41]. Collectively, our data suggest DPP-4 inhibition could suppress PDGF-BB-induced proliferation and migration of human PASMCs via PTEN-Akt-p38/ERK1/2 signaling pathway. However, the exact mechanism by which DPP-4 inhibition upregulates the expression of PTEN needs to be further investigated.

Apoptosis resistance of pulmonary vascular cells (ECs, PAMSCs, and adventitial fibroblasts) is another character of $\mathrm{PH}$ [42]. In our in vivo study, cell apoptosis around pulmonary arteries was inhibited by MCT, which was reversed by SG treatment. Due to the fact that few human PASMCs were undergoing apoptosis after quiesced for $48 \mathrm{~h}$, no remarkable anti-apoptotic effect was observed in PDGFBB-challenged human PASMCs, nevertheless, SG treatment significantly enhanced the apoptosis of human PASMCs. The PTEN-Akt-p38/ERK1/2 signaling pathway aforementioned has been well demonstrated to be involved in the regulation of cell apoptosis. It might also contribute to the pro-apoptotic effect of DPP-4i in human PASMCs.

In conclusion, our data in the present study imply that DPP-4 might be involved in the development of PH, and soluble DPP-4 in serum might be a diagnostic or prognostic marker for PH. By acting on PTEN-Akt-p38/ERK1/2 signaling pathway, DPP-4 inhibitors might be potential therapeutic drugs for $\mathrm{PH}$.

Acknowledgements This study was supported by the National Natural Science Foundation of China (No. 81273571), the Jiangsu Clinical Research Center for Respiratory Diseases Project under Grant BL2012012, the Jiangsu Province Scientific Research Innovation Project of University graduate students (JX22013361), and by a project funded by the Priority Academic Program Development of Jiangsu Higher Education Institutions (PAPD) (JX10231802).

\section{Compliance with ethical standards}

Conflict of interest The authors declare that they have no conflict of interest.

\section{References}

1. Tuder RM, Archer SL, Dorfmuller P, et al. Relevant issues in the pathology and pathobiology of pulmonary hypertension. J Am Coll Cardiol. 2013;62:D4-12. 
2. Lambeir AM, Durinx C, Scharpe S, et al. Dipeptidyl-peptidase IV from bench to bedside: an update on structural properties, functions, and clinical aspects of the enzyme DPP IV. Crit Rev Clin Lab Sci. 2003;40:209-94.

3. Thompson MA, Ohnuma K, Abe M, et al. CD26/dipeptidyl peptidase IV as a novel therapeutic target for cancer and immune disorders. Mini Rev Med Chem. 2007;7:253-73.

4. Xu J, Wang J, Cheng Y, et al. Glucagon-like peptide-1 mediates the protective effect of the dipeptidyl peptidase iv inhibitor on renal fibrosis via reducing the phenotypic conversion of renal microvascular cells in monocrotaline-treated rats. Biomed Res Int. 2018;2018:1864107.

5. Matheeussen V, Jungraithmayr W, De Meester I. Dipeptidyl peptidase 4 as a therapeutic target in ischemia/reperfusion injury. Pharmacol Ther. 2012;136:267-82.

6. West JW. Alcoholism: a general hospital meets the challenge. Md State Med J. 1976;25:73-76.

7. Panchapakesan U, Pollock C. The role of dipeptidyl peptidase-4 inhibitors in diabetic kidney disease. Front Immunol. 2015;6:443.

8. Takada S, Masaki Y, Kinugawa S, et al. Dipeptidyl peptidase-4 inhibitor improved exercise capacity and mitochondrial biogenesis in mice with heart failure via activation of glucagon-like peptide-1 receptor signalling. Cardiovasc Res. 2016;111:338-47.

9. Matsubara J, Sugiyama S, Sugamura K, et al. A dipeptidyl peptidase-4 inhibitor, des-fluoro-sitagliptin, improves endothelial function and reduces atherosclerotic lesion formation in apolipoprotein E-deficient mice. J Am Coll Cardiol. 2012;59:265-76.

10. Hirano T, Yamashita S, Takahashi M, et al. Anagliptin, a dipeptidyl peptidase-4 inhibitor, decreases macrophage infiltration and suppresses atherosclerosis in aortic and coronary arteries in cholesterol-fed rabbits. Metabolism . 2016;65:893-903.

11. Salheen SM, Panchapakesan U, Pollock CA, et al. The DPP-4 inhibitor linagliptin and the GLP-1 receptor agonist exendin-4 improve endothelium-dependent relaxation of rat mesenteric arteries in the presence of high glucose. Pharmacol Res. 2015;94:26-33.

12. Terawaki Y, Nomiyama T, Kawanami T, et al. Dipeptidyl peptidase-4 inhibitor linagliptin attenuates neointima formation after vascular injury. Cardiovasc Diabetol. 2014;13:154.

13. Ervinna N, Mita T, Yasunari E, et al. Anagliptin, a DPP-4 inhibitor, suppresses proliferation of vascular smooth muscles and monocyte inflammatory reaction and attenuates atherosclerosis in male apo E-deficient mice. Endocrinology . 2013;154:1260-70.

14. Liu L, Liu J, Wong WT, et al. Dipeptidyl peptidase 4 inhibitor sitagliptin protects endothelial function in hypertension through a glucagon-like peptide 1-dependent mechanism. Hypertension. 2012;60:833-41.

15. Wang JJ, Zuo XR, Xu J, et al. Evaluation and treatment of endoplasmic reticulum (ER) stress in right ventricular dysfunction during monocrotaline-induced rat pulmonary arterial hypertension. Cardiovasc Drugs Ther. 2016;30:587-98.

16. Wang J, Xu J, Zhao X, et al. Fasudil inhibits neutrophilendothelial cell interactions by regulating the expressions of GRP78 and BMPR2. Exp Cell Res. 2018;365:97-105.

17. Hirakawa H, Zempo H, Ogawa M, et al. A DPP-4 inhibitor suppresses fibrosis and inflammation on experimental autoimmune myocarditis in mice. PLOS One. 2015;10:e0119360.

18. Shirakawa J, Fujii H, Ohnuma K, et al. Diet-induced adipose tissue inflammation and liver steatosis are prevented by DPP-4 inhibition in diabetic mice. Diabetes. 2011;60:1246-57.

19. Alam MA, Chowdhury MRH, Jain P, et al. DPP-4 inhibitor sitagliptin prevents inflammation and oxidative stress of heart and kidney in two kidney and one clip (2K1C) rats. Diabetol Metab Syndr. 2015;7:107.

20. Ikedo T, Minami M, Kataoka H, et al. Dipeptidyl peptidase-4 inhibitor anagliptin prevents intracranial aneurysm growth by suppressing macrophage infiltration and activation. J Am Heart Assoc. 2017;6:e04777.

21. Dai Y, Dai D, Wang X, et al. DPP-4 inhibitors repress NLRP3 inflammasome and interleukin-1beta via GLP-1 receptor in macrophages through protein kinase $\mathrm{C}$ pathway. Cardiovasc Drugs Ther. 2014;28:425-32.

22. Zeisberg M, Zeisberg EM. Evidence for antifibrotic incretinindependent effects of the DPP-4 inhibitor linagliptin. Kidney Int. 2015;88:429-31.

23. Bostick B, Habibi J, Ma L, et al. Dipeptidyl peptidase inhibition prevents diastolic dysfunction and reduces myocardial fibrosis in a mouse model of Western diet induced obesity. Metabolism. 2014;63:1000-11.

24. Uchida T, Oda T, Matsubara $\mathrm{H}$, et al. Renoprotective effects of a dipeptidyl peptidase 4 inhibitor in a mouse model of progressive renal fibrosis. Ren Fail. 2017;39:340-9.

25. Takagaki Y, Koya D, Kanasaki K. Dipeptidyl peptidase-4 inhibition and renoprotection: the role of antifibrotic effects. Curr Opin Nephrol Hypertens. 2017;26:56-66.

26. Shi S, Srivastava SP, Kanasaki M, et al. Interactions of DPP-4 and integrin betal influences endothelial-to-mesenchymal transition. Kidney Int. 2015;88:479-89.

27. Shi S, Kanasaki K, Koya D. Linagliptin but not Sitagliptin inhibited transforming growth factor-beta2-induced endothelial DPP-4 activity and the endothelial-mesenchymal transition. Biochem Biophys Res Commun. 2016;471:184-90.

28. Good RB, Gilbane AJ, Trinder SL, et al. Endothelial to mesenchymal transition contributes to endothelial dysfunction in pulmonary arterial hypertension. Am J Pathol. 2015;185: 1850-8.

29. Rajkumar R, Konishi K, Richards TJ, et al. Genomewide RNA expression profiling in lung identifies distinct signatures in idiopathic pulmonary arterial hypertension and secondary pulmonary hypertension. Am J Physiol Heart Circ Physiol. 2010;298: H1235-48.

30. Tuder RM, Marecki JC, Richter A, et al. Pathology of pulmonary hypertension. Clin Chest Med. 2007;28:23-42.

31. Wronkowitz N, Gorgens SW, Romacho T, et al. Soluble DPP4 induces inflammation and proliferation of human smooth muscle cells via protease-activated receptor 2. Biochim Biophys Acta. 2014;1842:1613-21.

32. Kaji K, Yoshiji H, Ikenaka Y, et al. Dipeptidyl peptidase-4 inhibitor attenuates hepatic fibrosis via suppression of activated hepatic stellate cell in rats. $\mathrm{J}$ Gastroenterol. 2014;49:481-91.

33. Lim S, Choi SH, Shin H, et al. Effect of a dipeptidyl peptidase-IV inhibitor, des-fluoro-sitagliptin, on neointimal formation after balloon injury in rats. PLOS One. 2012;7:e35007.

34. Sweeney MD, Ayyadurai S, Zlokovic BV. Pericytes of the neurovascular unit: key functions and signaling pathways. Nat Neurosci. 2016;19:771-83.

35. Ogawa A, Firth AL, Smith KA, et al. PDGF enhances storeoperated $\mathrm{Ca} 2+$ entry by upregulating STIM1/Orai1 via activation of Akt/mTOR in human pulmonary arterial smooth muscle cells. Am J Physiol Cell Physiol. 2012;302:C405-11.

36. Ha D, Mester J, Eng C, et al. Pulmonary arterial hypertension in a patient with Cowden syndrome and the PTEN mutation. Pulm Circ. 2014;4:728-31.

37. Horita H, Furgeson SB, Ostriker A, et al. Selective inactivation of PTEN in smooth muscle cells synergizes with hypoxia to induce severe pulmonary hypertension. J Am Heart Assoc. 2013;2: e000188.

38. Worby CA, Dixon JE. PTEN. Annu Rev Biochem. 2014;83:641-69.

39. Teresi RE, Waite KA. PPARgamma, PTEN, and the fight against cancer. PPAR Res. 2008;2008:932632. 
40. Jomary C, Cullen J, Jones SE. Inactivation of the Akt survival pathway during photoreceptor apoptosis in the retinal degeneration mouse. Invest Ophthalmol Vis Sci. 2006;47:1620-9.

41. Jiao Y, Sun KK, Zhao L, et al. Suppression of human lung cancer cell proliferation and metastasis in vitro by the transducer of ErbB-2.1 (TOB1). Acta Pharmacol Sin. 2012;33:250-60.

42. Meloche J, Le Guen M, Potus F, et al. miR-223 reverses experimental pulmonary arterial hypertension. Am J Physiol Cell Physiol. 2015;309:C363-72. 